\title{
Horarios laborales de los progenitores y su incidencia en el rendimiento académico de alumnos de primaria. Efectos diferenciales del género
}

\author{
Ramon Cladellas Prosi, Antoni Castelló Tarridai, Mercè \\ Clariana Muntadai \& Mar Badia Martin \\ Universidad Autónoma de Barcelona, España
}

\section{Resumen}

Cada vez son más las mujeres que se incorporan al mercado laboral con lo que cada vez son más los hogares en que trabajan ambos progenitores. El objetivo de este trabajo era comprobar la incidencia de los horarios laborales de ambos progenitores, tratados de forma conjunta y por separado, en el rendimiento académico de alumnos de primaria en España. Para ello se contó con una muestra de 658 estudiantes (48\% niñas y 52\% niños, con una edad media de 8,69 años). Se administró un cuestionario a los padres para recabar información básica de aspectos sociodemográficos y familiares. Los resultados indican que las mejores notas, sobre todo en aquellas asignaturas en que se requiere un mayor esfuerzo cognitivo, se obtienen cuando al menos uno de los dos progenitores sigue un horario laboral estándar. Este resultado se ve acentuado cuando es la madre quien sigue un horario laboral estándar y no el padre.

\section{Palabras-clave}

Tipo horario; Horario laboral; Organización familiar; Rendimiento académico 


\section{Introducción}

El número de mujeres en el mundo laboral remunerado ha aumentado considerablemente desde la década de los 70 , lo que ha tenido como consecuencia un mayor número de hogares en que trabajan ambos progenitores (Cho \& Coulton, 2016; Saginak y Saginak, 2005), teniendo ello importantes repercusiones en la vida familiar. Otro aspecto a destacar son los horarios de trabajo en el mundo occidental, que a veces se acercan a lo que se ha denominado $7 \times 24$, ya que se admite como normal o habitual que uno pueda trabajar a cualquier hora del día, cualquier día de la semana, todos los días del año. Presser (2003) sugiere que trabajar fuera de lo denominado "horario estándar" (de las $9 \mathrm{~h}$ de la mañana a las $17 \mathrm{~h}$ de la tarde) es generalmente perjudicial para la vida familiar. Así, en 2004 , cerca del $18 \%$ de los trabajadores norteamericanos (unos 22 millones de personas) tenían horarios laborales considerados como no estándar, lo cual puede ser definido como un trabajo por turnos o con un horario fuera de lo que se consideraría como una jornada laboral regular de lunes a viernes (McMenamin, 2007). Este tipo de horarios son extensibles a la mayor parte de países desarrollados; en concreto, en Europa la prevalencia de estos horarios oscila entre un 14,5\% en Luxemburgo y un $29,4 \%$ en el Reino Unido. En España la prevalencia de estos horarios atípicos se sitúa alrededor del 15,7\% (Gracia \& Kalmijn, 2016).

Este tipo de horarios ha modificado la vida y organización familiar, afectando negativamente, no solo a la salud y bienestar de los trabajadores, sino también al desarrollo de sus hijos (Li et al., 2014). Algunos autores han analizado cómo este trabajo a todas horas influye en la vida académica de los alumnos de primaria. Han puesto de manifiesto que, por ejemplo, los hijos de padres con horario laboral nocturno o con cambios continuos en los turnos de trabajo obtienen notas más bajas en la escuela y presentan más problemas de conducta (Conger et al., 2002; Han, 2005).

En este estudio, utilizamos una muestra española para analizar la incidencia que tienen los padres por separado (madre con jornada laboral estándar y padre con jornada laboral no estándar; padre con jornada laboral estándar y madre con jornada laboral no estándar) o de forma conjunta (ambos progenitores con jornada laboral estándar y ambos progenitores con jornada laboral no estándar) en el rendimiento académico de sus hijos de primaria. Establecimos como jornada laboral estándar la franja de $8 \mathrm{~h}$ a $17 \mathrm{~h}$, 
teniendo en consideración lo aceptado por horario laboral estándar en un contexto español (Gracia \& Kalmijn, 2016; Gutiérrez-Domenech, 2010).

\section{Estado de la cuestión}

Como hemos dicho, la implicación de los padres en el bienestar y los resultados de los niños de primaria en la educación formal ha sido analizada con frecuencia (Lauer et al., 2006; Pierce, Bolt, \& Vandell, 2010). El estudio de dicha implicación puede abordarse desde dos aspectos (Vincent \& Neis, 2011). El primero hace referencia al tiempo que padres e hijos pasan juntos y es imprescindible para que se dé el segundo; y el segundo aspecto es la calidad de la interacción durante este tiempo de experiencias compartidas.

Diversos estudios previos demuestran que cuando aumenta la disponibilidad de los padres para estar con sus hijos (parental availability) el aprovechamiento escolar de los niños también aumenta significativamente. Por ejemplo, se ha puesto en evidencia que los hijos de los progenitores que tienen más tiempo para ayudarles en los deberes escolares obtienen mejores notas en la educación primaria, sobretodo en matemáticas y habilidad lectora, y también se adaptan mejor al entorno escolar (Ice \& Hoover-Dempsey, 2011; Koury \& Votruba-Drzal, 2014; Stacer \& Perucci, 2013; Sy, Gottfried, \& Gottfried, 2013).

Los motivos que determinan que los padres de los alumnos de primaria dediquen tiempo a estar con sus hijos son diversos. Siguiendo a Vincent y Neis (2011), se pueden clasificar en relación a algunos aspectos fácilmente objetivables, como los horarios laborales de los padres, las ocupaciones laborales en turnos a horas intempestivas, las largas horas pasadas en el transporte (commuting distance) entre el hogar y el lugar de trabajo, y el hecho de que la madre tenga un trabajo fuera de casa.

Los hijos de padres con horario laboral nocturno o con cambios continuos en los turnos de trabajo obtienen notas más bajas en la escuela y presentan más problemas de conducta (Conger et al., 2002; Han, 2005). En la misma línea, Hsueh y Yoshikawa (2007) demuestran que, controlando diversos aspectos de la vida familiar, como variables demográficas relativas a lugar de procedencia, nivel socioeconómico, inestabilidad laboral, salud mental de los padres y características del puesto de trabajo de los mismos, 
las ocupaciones parentales fuera de las horas habituales (de $8 \mathrm{~h}$ de la mañana a $4 \mathrm{~h}$ de la tarde en Estados Unidos, de lunes a viernes), combinadas con cambios frecuentes en los turnos de trabajo, se asocian a una disminución en la regularidad de los horarios de comidas y sueño en las familias, así como a un aumento en la presión a causa de la falta de tiempo que perciben los padres, dos características que redundan en las mencionadas notas más bajas y los problemas de comportamiento de los niños en la escuela. Sin embargo, no parece tratarse de una causa directa, ya que otras investigaciones ponen de relieve que la duración y la calidad del sueño de los niños están conectadas con su rendimiento en la escuela: pocas horas de sueño predicen bajo aprovechamiento (Cladellas, Chamarro, Badia, Oberst, \& Carbonell, 2011; El Sheikh, Buckhalt, Keller, Cummings, \& Acebo, 2007).

Por otra parte, se ha comprobado que, actualmente, los horarios de trabajo en algunos sectores, como los servicios, la banca o las nuevas tecnologías, pueden llegar a alargarse hasta 70-80 horas a la semana (Bunting, 2004; Lyonette \& Clark, 2009). Ya que la mayor parte de estos empleados son padres de ambos géneros (Sauvé, 2002), el tiempo que tienen para estar con sus hijos se reduce considerablemente (Vincent \& Neis, 2011). $Y$, cuando esto sucede, diversas investigaciones demuestran que los padres dejan de involucrarse en la educación formal de sus hijos, de modo que no revisan sus deberes, no siguen su progreso en el aprendizaje y no leen conjuntamente con ellos, lo que repercute en una disminución significativa en los resultados que los niños obtienen en la escuela (Barnes, Bryson, \& Smith, 2006; Lyonette \& Clark, 2009).

En cuanto a las horas de transporte, cabe comentar que la creciente presión y competencia en el mundo laboral también implica que cada vez sea más necesario aceptar trabajos fuera del lugar de residencia, con lo que las horas dedicadas a la movilidad aumentan. Por ejemplo, se ha comprobado que en una sola década se dobló el tiempo que se destina a acceder al lugar de trabajo (Pooley \& Turnbull, 1999) y, en el caso extremo de que uno o ambos progenitores trabajen fuera del país de residencia, se ha demostrado que la falta de contacto continuado con los hijos deteriora la relación familiar, disminuye el compromiso de los padres con la educación de sus hijos y también los resultados escolares de los mismos (Rogers, Theule, Ryan, Adams, \& Keating, 2009). 
En relación con el hecho de que la madre trabaje o no fuera de casa, se han publicado diversas consideraciones. Algún estudio ha informado de los efectos negativos del empleo materno sobre el desarrollo cognitivo de los niños, especialmente durante el primer año de vida (Han, Waldfogel, \& Brooks-Gunn, 2001). No obstante, otros autores han relacionado la ocupación laboral de la madre con un incremento en las capacidades cognitivas de los niños (Harvey, 1999). Otros arguyen que el hecho de que la influencia sea positiva o negativa se debe a las condiciones específicas del puesto de trabajo que ocupa la madre, como el sueldo que tiene, la complejidad del trabajo realizado y el tiempo que pasa fuera de casa, principalmente durante las horas en que los niños ya han vuelto del colegio (Smolensky \& Gootman, 2003). De esta manera, diferentes estudios (Han, Miller, \& Waldfogel, 2010; Wight, Raley, \& Bianchi, 2008) sugieren que las madres con horarios laborales no estándar pasan significativamente menos tiempo con sus hijos que aquellas otras madres con horarios laborales estándar.

Por último, autores como Han y Fox (2011) encontraron diferencias de género en el número de años que los progenitores habían estado trabajando en turnos de noche. Un mayor número de años de las madres en turnos de noche estaba significativamente relacionado con la obtención de peores notas de los alumnos en lectura y matemáticas. En contraste, el número de años que habían estado los padres trabajando en turnos de noche estaban significativamente asociados con un más alto rendimiento en matemáticas, aunque las diferencias eran mínimas. Estos resultados justifican una mayor investigación en relación con el posible efecto diferencial de los padres y madres en los horarios laborales no estándar.

Existen indicios de efectos diferenciales en la incidencia de la atención de los progenitores con los niños según el género de unos y otros. En concreto, Brooks-Gunn, Han, y Waldfogel (2002) afirman que la asociación entre el horario laboral de la madre tienen mayores incidencias cognitivas en los niños que en las niñas. En cualquier caso, el estudio está circunscrito al horario laboral cuando son bebés, por lo que no contempla la franja de edad estudiada en este trabajo.

Si bien existen estudios que ponen de manifiesto que este tipo de horarios tienen repercusiones directas en los resultados académicos de los adolescentes (Han \& Fox, 2011), como consecuencia del poco tiempo de que 
disponen los padres para supervisar y controlar las tareas que deben realizar los adolescentes fuera del colegio, todavía queda mucha investigación por realizar de este tema. En primer lugar, pocos estudios han investigado las consecuencias de los horarios labores no estándar en el rendimiento de alumnos de primaria en España que, como otros países del sur de Europa, se caracteriza por tener unas determinadas políticas de convivencia familiar (Lewis, 2009) y una tendencia por parte de ambos progenitores a cumplir largas jornadas laborales (Esping-Andersen, Boertien, Bonke, \& Gracia, 2013; Gracia \& Kalmijn, 2016). En segundo lugar, la mayoría de los estudios realizados analizan de forma conjunta el papel de los progenitores. Y, en tercer lugar, en ningún estudio se ha diferenciado la incidencia del horario no estándar entre asignaturas con fuerte carga cognitiva (Matemáticas, Lenguas y Idiomas) y con menor carga (Educación Física y Educación Artística). Así, pues, el objetivo de este trabajo es incidir en estos tres aspectos y analizar qué repercusión tiene en el rendimiento académico de niños entre 6 y 12 años, tanto conjuntamente como de forma separada por género.

\section{Método}

\section{Participantes}

Para construir la muestra de la investigación se hizo una primera selección de 37 centros educativos de primaria concertados de Cataluña (España). Dichos colegios provienen de: a) Barcelona ciudad, un 34\% de centros del total de la muestra; b) ciudades catalanas de más de 100.000 habitantes, un $33 \%$ de escuelas de la muestra; y c) ciudades o pueblos de Cataluña con menos de 100.000 habitantes, que constituyen un 33\% del total de centros educativos de la muestra. La selección de los centros fue encargada a los distintos centros territoriales de las provincias de Cataluña (Barcelona, Tarragona, Lérida y Gerona) y por medio de un muestreo aleatorio estratificado.

Para este estudio solo se tuvieron en cuenta colegios concertados a fin de controlar la variable socioeconómica de las familias, aspecto que se completó recopilando información sobre el tipo de trabajo de ambos progenitores, para garantizar de que al menos uno de los dos tuviera ingresos superiores a 30.000 euros, es decir, medio-altos. La muestra final estuvo for- 
mada por un total de 658 estudiantes (48\% niñas y 52\% niños, con una edad media de 8,69 años). Los criterios de inclusión fueron: que los padres de los alumnos seleccionados vivieran juntos en el domicilio familiar; que el tiempo de trayecto entre el colegio y el domicilio familiar no fuera superior a 30 minutos; que ambos cónyuges estuvieran activos laboralmente, ya sea por cuenta propia o ajena; que no tuvieran un trabajo por turnos; que el total de horas laborales semanales estuviera entre 35 y 45; que los niños seleccionados no realizaran más de una actividad extraescolar. Este último criterio se incluyó para garantizar que los niños y niñas de primaria estuvieran un mínimo de horas en casa durante los días laborales de la semana. Y, finalmente, también se consideró que los progenitores de cada familia seleccionada tuvieran estudios superiores, considerándose como tales a partir del nivel 5 de la Clasificación Nacional de Educación-2014 (CNED, 2014). En concreto, en el nivel 5, el 43\%; en el nivel 6, el 31\%; en el nivel 7, el 23\%; y en el nivel 8 , el $3 \%$.

Los citados criterios de inclusión se definieron para neutralizar el efecto de las variables socio-económicas, socio-culturales y de disponibilidad temporal. La variable calidad de la interacción se asumió como aleatoriamente distribuida entre los distintos grupos de horarios laborales. Por supuesto, el horario laboral determina la cantidad de tiempo de contacto entre los progenitores y los niños, aunque ello no implique que dicho tiempo se dedique a interacciones escolarmente favorecedoras. Dicha cualidad escapó del control en el diseño planteado, aunque puede asumirse como aleatorizada y, por ello, esperar un efecto de poca relevancia en los resultados o bien incidir en la dirección opuesta a las hipótesis planteadas.

\section{Instrumentos y material}

Se utilizó el mismo cuestionario empleado en otros estudios (Cladellas, Clariana, Gotzens, Badia, \& Dezcallar, 2015) para recabar información básica de aspectos sociodemográficos y familiares: tipo de trabajo, horario del trabajo del padre y de la madre, días y horas de la jornada laboral, y calificaciones académicas de los alumnos de primaria.

Una vez recopilada esta información, las variables estudiadas fueron:

(i) Tipo de horario. Esta variable se clasificó en dos grupos: horario laboral estándar de los padres vs horario laboral no estándar de los padres. 
Teniendo en cuenta el contexto cultural y los criterios empleados en otros estudios (Gracia \& Kalmijn, 2016; Gutiérrez-Domenech, 2010), se consideró que una jornada laboral estándar es aquella que se realiza de lunes a viernes, entre las $8 \mathrm{~h}$ de la mañana y las $17 \mathrm{~h}$ de la tarde. Esta variable se definió para cada progenitor.

(ii) Horario laboral de los progenitores. Teniendo en consideración la variable tipo de horario, se determinaron los siguientes cuatro grupos: grupo 1 - madre con jornada laboral estándar y padre con jornada laboral no estándar; grupo 2 - padre con jornada laboral estándar y madre con jornada laboral no estándar; grupo 3 - ambos progenitores con jornada laboral no estándar; grupo 4 - ambos progenitores con jornada laboral estándar.

(iii) Rendimiento académico. Se valora mediante la calificación obtenida por los alumnos en la última evaluación realizada en aquellas asignaturas que son comunes en el currículo de primaria: Matemáticas (MAT), Lenguas (LEN; que resulta de la nota media entre la calificación en Lengua castellana y Lengua catalana), Idiomas (ID), Educación Física (EF) y Educación Artística (EA). Para la conversión de las calificaciones en valores cuantitativos se utilizaron las siguientes equivalencias: suspenso $=2.5$; aprobado $=5.5$; bien $=6.5$; notable $=8$; sobresaliente $=9.5$. Se consideraron los valores medios de cada calificación.

\section{Procedimiento}

En una primera fase, el equipo de investigadores contactó con la dirección de los centros escolares con la intención de explicar el objetivo del trabajo, así como para informar de que se administraría un cuestionario a los padres de los alumnos seleccionados al azar.

La entrega y recogida de los cuestionarios se realizaba a través de la dirección de los centros, pues éstos eran los encargados de entregarlos a los niños y de recogerlos, una vez comprobaran que el cuestionario estaba debidamente cumplimentado. Para ello disponían de un tiempo máximo de tres semanas. Todos los participantes fueron informados y dieron su consentimiento para su inclusión en el estudio sin recibir a cambio ningún tipo de contraprestación.

Los cuestionarios que no cumplían los criterios de inclusión o no se recibieron dentro del plazo fijado fueron excluidos de la muestra. Así, por una u otra causa, se desestimaron un total de 1.648 cuestionarios. 


\section{Análisis de datos}

Se realizó un análisis multivariante de la Varianza (MANOVA) y un análisis univariado de la Varianza (ANOVA); en ambos casos la variable dependiente era la nota obtenida en las materias evaluadas; mientras que los factores fueron, para el MANOVA, el horario laboral de los progenitores (estándar o no estándar) y el género de los hijos; mientras que para el ANOVA, el horario laboral de los progenitores (cuatro grupos de combinaciones del horario del padre y de la madre). De todos los análisis efectuados se obtuvo el valor Eta al cuadrado parcial (np2) como medida del tamaño del efecto, considerando que una Eta al cuadrado parcial de .01 es pequeña, de .04 es moderada, y de .1 es grande (Huberty, 2002). Además se realizó un análisis de regresión lineal tomando como variables predictoras el número de horas de trabajo a la semana, la hora de finalización de la jornada laboral de ambos progenitores y el género de los hijos. Se tomaron como variables criterio sólo las que resultaron significativas en los análisis de la varianza. Los cálculos se realizaron con el paquete estadístico SPSS/PC+ (Versión 21.0) y los tests estadísticos fueron bilaterales con error tipo I variable al $5 \%$.

\section{Resultados}

\section{Descripción de la muestra}

En el $18.5 \%$ de los hogares de los niños seleccionados, ambos progenitores tenían horarios laborales no estándar, los cuales están en consonancia con los datos encontrados entre la población española (Gracia \& Kalmijn, 2016). El promedio de horas trabajadas semanalmente era de 40.55 para los padres y de 38.31 para las madres, parámetros que también se encuentran en línea a lo que ocurre en la población española. La hora media de finalización de la jornada laboral del padre era de una hora más tarde que la de la madre (18.17 vs 17.27). En promedio, la edad de los padres era tres años superior a la de las madres (40.2 vs 37.6).

La edad media de los niños era de 8.69 años, de los cuáles el 48\% eran niñas y el $52 \%$ niños. El $87 \%$ de los niños realizaban una actividad extraescolar, los niños y niñas restantes no realizaban ninguna. El promedio de las notas obtenidas en las diferentes materias analizadas oscilaba entre 7.28 y 8.01 . 
Se observan diferencias significativas en las notas de matemáticas y lenguas. Los niños en que al menos uno de sus padres sigue una jornada laboral estándar obtienen mejores notas que aquellos otros en que ninguno de los dos progenitores tiene una jornada laboral estándar (parte superior de la Tabla 1). Así mismo, también se observan diferencias significativas ligadas al género en las materias de Lenguas y Educación Artística, siempre favorables a las niñas (parte inferior de la Tabla 1). La interacción entre género y tipo de horario solamente se ha observado significativa en la materia de Educación Artística.

Tabla 1 - Análisis Multivariado de la varianza con tipo de horario (parte superior) y género (parte inferior) como variables independientes y notas académicas como variables dependientes. Se considera horario no estándar si al menos uno de los progenitores tiene este tipo de horario

\begin{tabular}{|c|c|c|c|c|c|c|}
\hline \multirow[b]{2}{*}{ Notas } & \multicolumn{2}{|c|}{ Tipo de horario } & \multirow[b]{2}{*}{$F$} & \multirow[b]{2}{*}{$P$} & \multirow[b]{2}{*}{ пр2 } & \multirow[b]{2}{*}{$\begin{array}{l}\text { Potencia } \\
\text { Observa- } \\
\quad \text { da }\end{array}$} \\
\hline & $\begin{array}{l}\text { Horario } \\
\text { estándar } \\
(n=480)\end{array}$ & $\begin{array}{l}\text { Horario no } \\
\text { estándar } \\
(n=178)\end{array}$ & & & & \\
\hline Matemáticas & $7.58 \pm 1.94$ & $7.22 \pm 1.72$ & 4.63 & .032 & .007 & .58 \\
\hline Lenguas & $7.36 \pm 1.59$ & $7.06 \pm 1.56$ & 4.78 & .029 & .007 & .59 \\
\hline Idiomas & $7.39 \pm 1.82$ & $7.13 \pm 1.86$ & 2.56 & .110 & .004 & .36 \\
\hline Educación Física & $8.05 \pm 1.34$ & $7.92 \pm 1.29$ & 1.28 & .259 & .002 & .20 \\
\hline \multirow[t]{2}{*}{$\begin{array}{l}\text { Educación Artísti- } \\
\text { ca }\end{array}$} & $7.73 \pm 1.45$ & $7.61 \pm 1.32$ & 1.11 & .293 & .002 & .18 \\
\hline & \multicolumn{2}{|c|}{ Género } & & & & \\
\hline Notas & $\begin{array}{l}\text { Niños } \\
(n=343)\end{array}$ & $\begin{array}{l}\text { Niñas } \\
(n=315)\end{array}$ & $F$ & $P$ & np2 & $\begin{array}{c}\text { Potencia } \\
\text { Observa- } \\
\text { da }\end{array}$ \\
\hline Matemáticas & $7.58 \pm 1.89$ & $7.39 \pm 1.90$ & 0.38 & .540 & .001 & .09 \\
\hline Lenguas & $7.15 \pm 1.51$ & $7.42 \pm 1.66$ & 8.14 & .004 & .012 & .81 \\
\hline Idiomas & $7.30 \pm 1.83$ & $7.34 \pm 1.83$ & 0.91 & .340 & .001 & .16 \\
\hline Educación Física & $8.03 \pm 1.34$ & $8.00 \pm 1.32$ & 0.02 & .880 & .000 & .05 \\
\hline $\begin{array}{l}\text { Educación Artísti- } \\
\text { ca }\end{array}$ & $7.48 \pm 1.44$ & $7.94 \pm 1.36$ & 23.12 & .000 & .034 & 1.00 \\
\hline
\end{tabular}


Tabla 2 - Análisis de la varianza con grupos de horario laboral de los padres como variable independiente y notas académicas como variables dependientes

\begin{tabular}{|c|c|c|c|c|c|c|c|c|}
\hline \multirow[b]{2}{*}{ Notas } & \multicolumn{4}{|c|}{ Horario laboral de los padres } & \multirow[b]{2}{*}{$\boldsymbol{F}$} & \multirow[b]{2}{*}{$\boldsymbol{P}$} & \multirow[b]{2}{*}{ np2 } & \multirow[b]{2}{*}{$\begin{array}{c}\text { Potencia } \\
\text { observada }\end{array}$} \\
\hline & $\begin{array}{l}\text { Grupo } 1 \\
(n=206)\end{array}$ & $\begin{array}{c}\text { Grupo } 2 \\
(n=152)\end{array}$ & $\begin{array}{c}\text { Grupo } 3 \\
(n=178)\end{array}$ & $\begin{array}{c}\text { Grupo } 4 \\
(n=122)\end{array}$ & & & & \\
\hline Matemáticas & $7.76 \pm 1.97$ & $7.10 \pm 1.93$ & $7.22 \pm 1.72$ & $7.91 \pm 1.80$ & 6.86 & .000 & .030 & .98 \\
\hline Lenguas & $7.46 \pm 1.69$ & $6.99 \pm 1.54$ & $7.06 \pm 1.56$ & $7.66 \pm 1.39$ & 6.22 & .000 & .028 & .96 \\
\hline Idiomas & $7.48 \pm 1.84$ & $7.05 \pm 1.79$ & $7.13 \pm 1.86$ & $7.66 \pm 1.75$ & 3.76 & .011 & .017 & .81 \\
\hline Educación Física & $8.07 \pm 1.20$ & $7.93 \pm 1.34$ & $7.92 \pm 1.29$ & $8.16 \pm 1.56$ & 1.14 & .333 & .005 & .31 \\
\hline $\begin{array}{l}\text { Educación Artísti- } \\
\text { ca }\end{array}$ & $7.68 \pm 1.42$ & $7.57 \pm 1.52$ & $7.61 \pm 1.32$ & $8.00 \pm 1.39$ & 2.49 & .059 & .011 & 62 \\
\hline
\end{tabular}

La Tabla 2 muestra la media y desviación estándar obtenida para cada grupo. Se encontraron diferencias significativas globales en todas las asignaturas menos en Educación Física y Educación Artística. De manera concreta, los test post hoc indican que en las asignaturas de Matemáticas y Lenguas los grupos 1 y 4 difieren significativamente $(P<.05)$ de los grupos 2 y 3.

Tabla 3 - Análisis de regresión lineal tomando como variable criterio la puntuación obtenida en Matemáticas y como variables predictoras: el número de horas de trabajo a la semana, la hora de finalización de la jornada labo-ral de ambos progenitores y el género de los hijos

\begin{tabular}{llccc}
\hline & Predictores & Beta & Sig. & $\mathrm{R}^{2}$ \\
\hline Modelo 1 & Hora finalización jornada laboral madre & -.117 & .000 & .035 \\
\hline \multirow{2}{*}{ Modelo 2} & Hora finalización jornada laboral madre & -.108 & .000 & \\
& Horas trabajo madre & -.046 & .006 & .046 \\
\hline
\end{tabular}


Tabla 4 - Análisis de regresión lineal tomando como variable criterio la puntuación obtenida en Lenguas y como variables predictoras: el número de horas de trabajo a la semana, la hora de finalización de la jornada laboral de ambos progenitores y el género de los hijos

\begin{tabular}{llccc}
\hline & Predictores & Beta & Sig. & $\mathrm{R}^{2}$ \\
\hline Modelo 1 & Hora finalización jornada laboral madre & -.109 & .000 & .040 \\
\hline Modelo 2 & Hora finalización jornada laboral madre & -.195 & .000 & \\
& Género & .096 & .012 & .045 \\
\hline
\end{tabular}

Tabla 5 - Análisis de regresión lineal tomando como variable criterio la puntuación obtenida en Educación Artística y como variables predictoras: el número de horas de trabajo a la semana, la hora de finalización de la jornada laboral de ambos progenitores y el género de los hijos

\begin{tabular}{llccc}
\hline & Predictores & Beta & Sig. & $\mathrm{R}^{2}$ \\
\hline Modelo 1 & Género & .163 & .000 & .027 \\
\hline Modelo 2 & Género & .161 & .000 & \\
& Horas trabajo madre & -.137 & .000 & .045 \\
\hline Modelo 3 & Género & .168 & .000 & \\
& Horas trabajo madre & -.105 & .008 & \\
& Hora finalización jornada laboral madre & -.104 & .009 & .055 \\
\hline
\end{tabular}

El análisis de regresión lineal muestra que la variable que mejor predice el rendimiento académico en Matemáticas, Lenguas y Educación Artística es la hora de finalización de la jornada laboral de la madre, añadiéndose, en el caso de las Matemáticas, la variable horas de trabajo de la madre; mientras que en Lenguas y Educación Artística aparece la variable género. A pesar de la significación estadística del efecto, la cantidad de varianza explicada, tal como indican los índices R2, es muy moderada: con un máximo de $5,5 \%$ de varianza explicada.

\section{Discusión}

Los resultados indican que las mejores notas en Matemáticas y Lenguas, es decir, en aquellas asignaturas que requieren un mayor esfuerzo 
cognitivo, se obtienen entre los grupos en que al menos uno de los dos progenitores sigue un horario laboral estándar. Este resultado se produce en el caso en que es la madre la que sigue un horario laboral estándar y no el padre, tal como indican los análisis de la regresión.

La interpretación de estos resultados no es, ni mucho menos, directa, ya que confluyen numerosos aspectos explicativos que no han podido ser controlados por el diseño de la investigación, a pesar que sí que pueden considerarse como aleatorizados, o bien de efecto contrario al expuesto en las hipótesis. Por ejemplo, tener un horario laboral estándar no es garantía que se destine el tiempo disponible a los hijos, pudiendo dicho tiempo dedicarse a otras actividades que no impliquen a los hijos (como deporte $u$ otras formas de ocio adulto). Sin embargo, los efectos de estas variables actuarían reduciendo los efectos observados, los cuales han sido significativos, aunque moderados.

No son evidentes los efectos causales atribuibles directamente al género, ya que son diversas las explicaciones que podrían darse a estos resultados. Quizá la más obvia es que la ordenación de las actividades domésticas, y particularmente supervisión de las actividades de los hijos, parece seguir siendo una parte del rol materno más que del paterno (Maume \& Sebastian, 2012). En este sentido, los grupos en los cuales las madres disponen de un horario regular dan la oportunidad de que sean muchas las madres que desempeñan este rol. La posibilidad de que sea indistinto cuál de los progenitores disfruta de un horario estándar queda descartada al presentar el grupo 2, en el cual era el padre quien tenía dicho horario, los peores rendimientos en bastantes materias, incluso más bajo que el grupo 3 , en el cual ambos progenitores tienen horarios irregulares.

Incluso bajo la aceptación de la anterior diferencia de roles, los efectos causales seguirían disponiendo de dos vías explicativas distintas: en primer lugar, los efectos generales de una vida más ordenada y, de manera particular, de unos hábitos de sueño más saludables. Como ya ha sido mencionado en la introducción, los hábitos de comer y dormir también han demostrado estar relacionados con el aprovechamiento académico en niños de primaria (El Sheikh et al., 2007). Es lógico pensar que dichos hábitos dependen en gran parte del horario de trabajo de los progenitores, así como de sus horas de llegada a casa por la tarde/noche y del tiempo que pasan con 
sus hijos en los días laborables. A la influencia genérica de los horarios laborales del padre y de la madre sobre el aprovechamiento escolar de los alumnos de primaria, hay que añadir, tal y como señalan Hsueh y Yoshikawa (2007), otros aspectos también de tipo familiar, como son las actitudes y creencias de los padres hacia la escuela, las condiciones laborales de los progenitores, el contacto de los niños con otros familiares y/o cuidadores, y las actividades extraescolares en que los niños participan. En un trabajo previo (Cladellas, Clariana, Badia, \& Gotzens, 2013) se demostraba que un número moderado de horas de actividades extraescolares contribuye a mejorar las notas académicas, pero que, sobrepasado este número ideal, los efectos de un exceso de horas pasadas fuera de casa durante los días laborables cuando los niños han finalizado su jornada escolar son claramente perjudiciales. Esta última variable sí que ha sido controlada en los participantes del estudio, excluyendo aquellos casos que realizaban más de una actividad extraescolar.

Integrando estos resultados, puede contemplarse un componente actitudinal central en el cual los progenitores adoptan personalmente un rol activo en la educación de sus hijos, evitando delegarla completamente a agentes externos, sean en la escolarización reglada o en ac-ividades extraescolares. $Y$ es en este tipo de actitudes en las que los resultados obtenidos en el presente estudio parecen aportar cierta luz: parecen estar mucho más consolidadas en las madres que no en los padres. La explicación es sólo tentativa, puesto que no se ha medido directamente este tipo de actitud. Sin embargo, los resultados son claros en lo que implica el efecto diferencial de las madres que, por motivos sociológicos, se les puede atribuir este tipo de actitudes en una mayor medida. Por ello, de la combinación de disponibilidad de tiempo y una mayor probabilidad de que presenten estas actitudes resulta una explicación verosímil de los resultados observados.

En segundo lugar, el efecto de una supervisión directa de las actividades escolares realizadas en casa - los deberes - de manera que simplemente se acumularía una mayor cantidad de tiempo dedicado al aprendizaje académico, cuya relación con el rendimiento ha sido ampliamente demostrada (Pierce et al., 2010; Vincent \& Neis, 2011). Esta relación se hace especialmente notoria en el caso de las asignaturas de mayor demanda cognitiva, en la que la automatización de procesos es muy 
importante, pero habitualmente tediosa. En cambio, en materias como la Educación Física, las actividades afines que pueden facilitar la automatización motora no suelen tener formato de deberes, sino de juegos o deportes, a la vez que suelen ser más atractivos para buena parte de la población escolar de primaria.

De nuevo, no se ha medido directamente la implicación en los deberes filiales de padres y madres, particularmente de estas últimas, aunque se puede aplicar el mismo razonamiento probabilístico que se ha expresado más arriba.

Por supuesto, ambos efectos pueden producirse simultáneamente, ya que no son incompatibles, razón por la cual no es posible distinguir el uno del otro en este cuerpo de datos. En este sentido, la explicación planteada concuerda con Vincent y Neis (2011) cuando afirman que "parental work schedules exert an impact on parental involvement in children's education and parent child attachment quality through their effect on the physical and emotional availability of parents" (p. 459), y nuestros datos permiten ampliar esta aseveración remarcando la importancia del rol de la madre, que ha resultado ser diferencial al del padre en nuestro estudio. Las explicaciones de carácter emocional tampoco pueden ser descartadas, aunque parten de un escaso soporte empírico en la literatura antecedente.

En cualquier caso, como sucede siempre que se investigan variables como el género, es muy importante tomar amplias precauciones en la explicación de los resultados y, muy particularmente, en su divulgación. Una cuestión es el dato empírico obtenido y otra, muy distinta, su interpretación. Nótese que la consideración de este efecto como ligado al género insinúa una naturaleza estructural, biológica, del mismo. Por ello, sería poco susceptible de modificación u optimización. En cambio, si se vincula a las actitudes parentales, apelando a un mayor arraigo de las mismas entre las madres - lo cual es la conclusión más probable -, se está considerando un efecto social que es susceptible de modificación.

El género de los hijos ha aparecido también como una variable que, por sí misma, explica parte de las diferencias en el rendimiento. Este efecto ha sido especialmente visible en Lenguas y Educación Artística: en ambos casos las chicas obtienen mejores resultados que los chicos. Sin embargo, en la última de estas materias se pone de manifiesto una interacción entre el tipo 
de horario y el género, en la cual los chicos se ven favorecidos por el horario estándar de sus madres, mientras que las chicas no (de hecho, rinden algo peor que cuando las madres tienen un horario no estándar). Estos resultados serían acordes a los encontrados por Brooks-Gunn et al. (2002).

A la luz de los resultados que ahora presentamos, de lo que se trata en primera instancia es de aumentar el tiempo conjunto de que disponen padres e hijos durante la semana, y principalmente de ampliar el tiempo que la madre pasa con los niños cuando estos salen del colegio. Además de esta consideración, nuestro trabajo también sirve para poner de manifiesto la relevancia de los hábitos y del día a día en la vida de los niños, pues precisamente esto es lo que hemos estudiado, cómo afecta el tiempo que regularmente, cada día de la semana, el padre y la madre pasan con sus hijos.

Colateralmente, se debería de considerar una racionalización de los horarios laborales españoles. En contra de lo que se observa en los países del centro de Europa, los horarios españoles, en su mayor parte, se corresponden a horarios no estándar; ello explicaría el poco contacto que mantienen los padres de los niños y, consecuentemente, los efectos negativos en el rendimiento académico de éstos. Los matices atribuibles al género de los hijos existen, a pesar de que parecen secundarios en comparación con los que son producidos por el tipo de horario. Finalmente, cabe insistir en que el horario aporta la oportunidad de plantear una interacción favorecedora del rendimiento académico, pero no la causa. Solamente con las actitudes adecuadas y la implicación efectiva en este tipo de actividades se consigue el incremento del aprovechamiento escolar.

\section{Referências}

Barnes, M., Bryson, C., \& Smith, R. (2006). Working atypical hours: What happens to "family life" ? London: National Centre for Social Research.

Brooks-Gunn, J., Han, W. J., \& Waldfogel, J. (2002). Maternal employment and child cognitive outcomes in the first three years of life: The NICHD study of Early Child Care. Child Development, 73, 1052-1072.

Bunting, M. (2004). Willing slaves: How the overwork culture is ruling our lives. New York, NY: Harper Collins. 
Cho, Y., \& Coulton, C. J. (2016). The effects of parental nonstandard work schedules on adolescents' academic achievement in dual-earner households in South Korea. Child Indicators Research, 9, 193-212. doi: 10.1007/s12187-015-9308-4

Cladellas, R., Chamarro, A., Badia, M., Oberst, U., \& Carbonell, X. (2011). Efectos de las horas y los hábitos de sueño en el rendimiento académico de niños de 6 y 7 años: Un estudio preliminar. Cultura y Educación, 23(1),119-128.

Cladellas, R., Clariana, M., Badia, M., \& Gotzens, C. (2013). Actividades extraescolares y rendimiento académico en alumnos de primaria. European Journal of Investigation in Health, Psychology and Education, 3(2), 87-97.

Cladellas, R., Clariana, M., Gotzens, C., Badia, M., \& Dezcallar, T. (2015). Patrones de descanso, actividades físico-deportivas extraescolares y rendimiento académico en niños y niñas de primaria. Revista de Psicología del Deporte, 24(1), 53-59.

Clasificación Nacional de Educación [CNED] (2014). Instituto Nacional de Estadística. Madrid. Extraido el 27 de Julio de 2016 desde http://www.ine.es/jaxi/menu.do ?type=pcaxis\&path= \%2Ft40\%2Fcned 14\%2F\& file=inebase \&L=0

Conger, R. D., Wallace, L., Sun, Y., Simons, R. L., McLloyd, V., \& Brody, G. H. (2002). Economic pressure in African American families: A replication and extension of the family stress model. Developmental Psychology, 38, 179-193. doi: 10.1037/0012-1649.38.2.179

El-Sheikh, M., Buckhalt, J. A., Keller, P. S., Cummings, E. M., \& Acebo, C. (2007). Child emotional insecurity and academic achievement: The role of sleep disruptions. Journal of Family Psychology, 21(1), 29-38. doi: 10.1037/0893-3200.21.1.29

Esping-Andersen, G., Boertien, D., Bonke, J., \& Gracia, P. (2013). Couple specialization in multiple equiliria. European Sociological Review, 29(6), 1280-1294. doi:10.1093/esr/jct004

Gracia, P., \& Kalmijn, M. (2016). Parents' family time and work schedules: The split-shift schedule in Spain. Journal of Marriage and Family, 78, 401-415. doi:10.1111/jomf.12270

Gutiérrez-Domenech, M. (2010). Parental employment and time with children in Spain. Review of Economics of the Household, 8(3), 371-391. doi:10.1007/s11150-0109096-z

Han, W. J. (2005). Maternal nonstandard work schedules and child cognitive outcomes. Child Development, 76, 137-154. doi: 10.1111/j.1467-8624.2005.00835.x

Han, W. J., \& Fox, L. E. (2011). Parental work schedules and children's cognitive trajectories. Journal of Marriage and Family, 73(5), 962-980. doi:10.1111/j.17413737.2011.00862.x

Han, W. J., Miller, D. P., \& Waldfogel, J. (2010). Parental work schedules and adolescent risky behaviors. Developmental Psychology, 46(5), 1245-1267.

Han, W. J., Waldfogel, J., \& Brooks-Gunn, J. (2001). The effects of early maternal employment on later cognitive and behavioral outcomes. Journal of Marriage and the Family, 63, 336 354. doi: 10.1111/j.1741-3737.2001.00336.x

Harvey, E. (1999). Short-term and long-term effects of parental employment on children of the National Longitudinal Survey of Youth. Developmental Psychology, 35, 445-459. doi: 10.1037/0012-1649.35.2.445 
Hsueh, J., \& Yoshikawa, H. (2007). Working nonstandard schedules and variable shifts in low-income families: Associations with parental psychological well-being, family functioning, and child well-being. Developmental Psychology, 43(3), 620632. doi: 10.1037/0012-1649.43.3.620

Huberty, C. J. (2002). A history of effect sizes indices. Educational and Psychological Measurement, 62, 227-240.

Ice, C. L., \& Hoover-Dempsey, K. V. (2011). Linking parental motivations for involvement and student proximal achievement outcomes in homeschooling and public schooling settings. Education and Urban Society, 43(3), 339-369. doi: $10.1177 / 0013124510380418$

Koury, A. S., \& Votruba-Drzal, E. (2014). School readiness of children from immigrant families: Contributions of region of origin, home, and childcare. Journal of Educational Psychology, 106(1), 268-288. doi: 10.1037/a0034374

Lauer, P. A., Akiba, M., Wilkerson, S. B., Apthorp, H. S., Snow, D., \& Martin-Glenn, M. (2006). Out-of-school time programs: A meta-analysis of effects for at risk students. Review of Educational Research, 76, 275-313.

Lewis, J. (2009). Work-family balance, gender and policy. Cheltenham, UK: Elgar.

Li, J., Johnson, S. E., Han, W. J., Andrews, S., Kendall, G., Strazdins, L., \& Dockery, A. (2014). Parents' nonstandard work schedules and child well-being: A critical review of the literature. The Journal of Primary Prevention, 35(1), 53-73.

Lyonette, C., \& Clark, M. (2009). Unsocial hours: Unsocial families? Working time and family well being. Cambridge, UK: Relationships Foundation.

Maume, D. J., \& Sebastian, R. A. (2012). Gender, nonstandard work schedules, and marital quality. Journal of Family and Economic Issues, 33, 477-490.

McMenamin, T. M. (2007). A time to work: Recent trends in shift work and flexible schedules. Monthly Labor Review, 130, 3-15.

Pierce, K. M., Bolt, D. M., \& Vandell, D. L. (2010). Specific features of after-school program quality: Associations with children's functioning in middle childhood. American Journal of Community Psychology, 45, 381-393.

Pooley, C., \& Turnbull, J. (1999). The journey to work: A century of change. Area, 31, 281292.

Presser, H. B. (2003). Working in a 24/7 economy: Challenges for American families. New York, NY: Russell Sage Foundation.

Rogers, M., Theule, J., Ryan, B., Adams, G., \& Keating, L. (2009). Parental involvement and children's school achievement: Evidence for mediating processes. Canadian Journal of School Psychology, 24, 34-57.

Saginak, K. A., \& Saginak, M. A. (2005). Balancing work and family: Equity, gender, and marital satisfaction. The Family Journal, 13(2), 162-166.

Sauvé, R. (2002). Connection. Tracking the links between jobs and family. Contemporary Family Trends, Occasional Paper. Ottawa, CA: The Vanier Institute of the Family.

Smolensky, E., \& Gootman, J. (2003). Working families and growing kids: Caring for children and adolescents. Washington, DC: National Academies Press. 
Stacer, M. J., \& Perucci, R. (2013). Parental involvement with children at school, home, and community. Journal of Family and Economic Issues, 34(3), 340-354. doi:10.1007/s10834-012-9335-y

Sy, S. R., Gottfried, A. W., \& Gottfried, A. E. (2013). A transactional model of parental involvement and children's achievement from early childhood through adolescence. Parenting: Science and Practice, 13(2), 133-152. doi: $10.1080 / 15295192.2012 .709155$

Vincent, C. D., \& Neis, B. L. (2011). Work and family life: Parental work schedules and child academic achievement. Community, Work \& Family, 14(4), 449-468. doi: 10.1080/13668803.2010.535664

Wight, V. R., Raley, S. B., \& Bianchi, S. M. (2008). Time for children, one's spouse and one-self among parents who work nonstandard hours. Social Forces, 87(1), 243-271. 


\section{WORKING HOURS OF THE PARENTS AND THEIR IMPACT ON THE ACADEMIC PERFORMANCE OF PRIMARY SCHOOL STUDENTS. DIFFERENTIAL EFFECTS OF GENDER}

\section{Abstract}

There is an increasing number of women that develop full time jobs, something that causes a higher number of homes with both parents working. The goal of this research consisted in testing how parents' work schedule, considered individually and jointly, affects school performance in primary compulsory education students in Spain. The sample consisted of 658 students (48\% girls and $52 \%$ boys; mean age of 8,69 years). A questionnaire was administered to parents in order to gather demographic and family information. Results show that best marks, particularly in those subjects that are more cognitively demanding, are associated to those cases where at least one of the parents has a standard work schedule, being the maximum difference observed when is the mother who has the standard schedule.

Keywords

Standard schedule; Work schedule; Family organisation; School achievement

\section{HORÁRIOS LABORAIS DOS PROGENITORES E INCIDÊNCIA NO DESEMPENHO ACADÉMICO DOS ALUNOS DO $1^{\circ}$ CICLO DO ENSINO BÁSICO. EFEITOS DIFERENCIAIS DO GÉNERO}

\section{Resumo}

São cada vez mais as mulheres que se incorporam no mercado laboral, o que faz com que sejam cada vez mais as famílias em que ambos os progenitores trabalham. Este artigo tem por objetivo comprovar o impacto dos horários laborais dos pais, tratados em conjunto ou em separado, no rendimento académico de alunos do $1^{\circ}$ ciclo do ensino básico em Espanha. Para isso, considerou-se uma amostra de 658 alunos (48\% meninas e 52\% rapazes, 
com uma média de idades de 8,69 anos). Foi aplicado um questionário aos pais para recolher informação elementar sobre aspectos sociodemográficos e familiares. Os resultados indicam que as melhores classificações, sobretudo nas áreas curriculares que requerem maior esforço cognitivo, se obtêm quando pelo menos um dos dois progenitores segue um horario laboral regular. Estes resultados acentuam-se quanto é a mãe que segue um horário laboral standard e não o pai.

Palavras-chave

Tipo de horário; Horário laboral; Organização familiar; Rendimento académico

Recebido em junho/2016

Aceite para publicação em setembro/2016

i Departamento de Psicología Básica, Evolutiva y de la Educación, Universidad Autónoma de Barcelona, España.

Toda a correspondência relativa a este artigo deve ser enviada para: Ramon Cladellas Pros, Departamento de Psicología Básica, Evolutiva y de la Educación, Universidad Autónoma de Barcelona, 08193 Barcelona (España). E-mail: ramon.cladellas@uab.es 